\title{
Doctors repurposing medical devices and apps for COVID-19
}

- Cite as: CMAJ 2021 March 15;193:E386-7. doi: 10.1503/cmaj.1095927

Posted on cmajnews.com on February 26, 2021

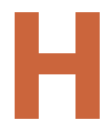

ealth care workers worldwide have risen to the challenges of the coronavirus disease 2019 (COVID-19) pandemic with creative solutions, from 3D-printing face shields, ventilator parts and nasopharyngeal swabs to repurposing home breathing machines as makeshift ventilators. Physicians and researchers in Canada are adapting health technologies to triage and monitor patients with COVID-19 too.

When the pandemic hit, Dr. Anthony Tang of the Cardiac Arrhythmia Network of Canada was working on an e-health platform for patients with arrhythmias, including those with implantable cardiac devices. Patients with these devices require regular follow-up, and the platform, called VIRTUES, allows them to monitor how their device is functioning and other health information.

With the pandemic delaying a trial of the platform, Tang says he couldn't "waste time doing nothing." So he and his colleagues adapted the technology to monitor patients with COVID-19 who are isolating at home.

Patients can use an app to log their symptoms, including oxygen saturation and pulse rate, and communicate with their care team to avoid unnecessary visits to emergency departments and clinics. Meanwhile, clinicians can review trends in their patients' recoveries to help identify those that may require hospitalization.

London Health Sciences Centre in Ontario launched a pilot of the platform in the fall last year, and since then, more than 300 patients in London, Ottawa, Kitchener and Waterloo have used the tool. While an evaluation of the platform is ongoing, Tang says it appears to be working well. "There have been several patients who have avoided hospitalization

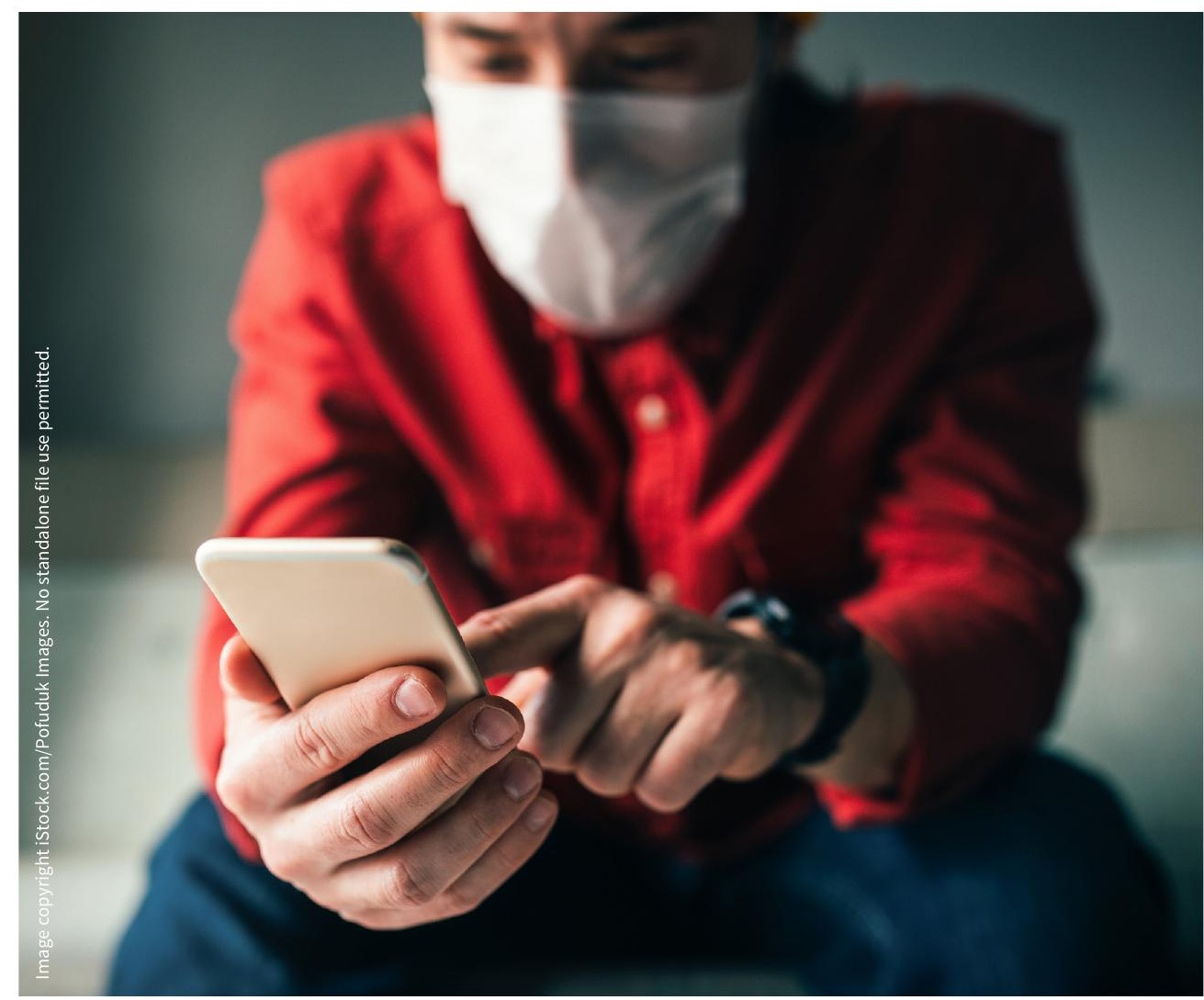

Canadian researchers are experimenting with new ways to triage and monitor patients with COVID-19.

with the aid of this monitoring and, alternatively, patients have been brought into hospital who needed more care."

Another team in Manitoba is developing a tool to triage patients with COVID-19 based on the sound of their breathing.

Zahra Moussavi, director of biomedical engineering at the University of Manitoba, studies acoustic technologies that have been used for years to diagnose a wide range of conditions, from asthma to infectious diseases. When COVID-19 emerged, Moussavi thought the same technologies could help triage suspected cases quickly.
Her team is working on a "respiratory system triage" app that records and analyzes a person's breathing to identify potential cases of COVID-19. Users also fill out a baseline questionnaire to account for other conditions that affect breathing sounds like chronic obstructive pulmonary disease, asthma, and heart failure.

According to Moussavi, "the goal is to develop a quick monitoring app so that within 30 seconds it can tell if a person is safe to enter a workspace." While she doesn't expect the app to replace other COVID-19 tests, it could offer a more reliable 
alternative to temperature checks - a widely used screening tool that some have criticized as ineffective pandemic theatre. Moussavi notes the app will need to achieve an accuracy rate of at least $70 \%$ to meet the bar for "good triage."

Similar work is underway in the United States. Scientists at the Massachusetts Institute of Health in Boston recently found that an artificial intelligence model could accurately diagnose COVID-19 based on a person's cough with a sensitivity of $98.5 \%$ and a specificity of $94.2 \%$. In asymptomatic people who were asked to force a cough, the program diagnosed COVID-19 with $100 \%$ sensitivity and $83.2 \%$ specificity.

According to the researchers, the technology could be used for "daily screening of students, workers, and the public as schools, jobs, and transport reopen, or for pool testing to quickly alert of outbreaks in groups."

However, as the second wave of the pandemic ebbs, Moussavi says recruiting enough people in Canada to train such a tool will be difficult and securing ethics approval delayed the start of her project.

Moussavi is also working with Azadeh Yadollahi of the University Health Network in Toronto on an app for monitoring patients with COVID-19. They hope to use computerized analysis of respiratory sounds to establish objective criteria for assessment. The app they're working on uses recordings of a patient's breathing together with other measures, such as blood pressure and oxygen saturation readings, to track recovery from COVID-19.

Yadollahi has previously developed wearable medical devices that can measure respiration and heart rate. She hopes that, by adapting these tools for COVID19 , they can help manage outbreaks in higher risk groups, such as people experiencing homelessness. According to Yadollahi, with the right tools to manage patients remotely, "we can take a lot of pressure off the health care system and caregivers."

\section{Dr. Maeve Gamble, London, Ont.}

Content licence: This is an Open Access article distributed in accordance with the terms of the Creative Commons Attribution (CC BY-NC-ND 4.0) licence, which permits use, distribution and reproduction in any medium, provided that the original publication is properly cited, the use is noncommercial (i.e., research or educational use), and no modifications or adaptations are made. See: https://creativecommons.org/ licenses/by-nc-nd/4.0/ 